\title{
Serum Fatty Acids Chemical Characterization after Prolonged Exposure to a Vegan Diet
}

\author{
Alan Espinosa-Marrón ${ }^{1}$, Hugo Laviada-Molina ${ }^{1}$, Angélica Moreno-Enríquez ${ }^{1}$, \\ Irving F. Sosa-Crespo ${ }^{1}$, Fernanda Molina-Segui ${ }^{1}$, María Fernanda Villaseñor-Espinosa², \\ José Antonio Ciau-Mendoza ${ }^{3}$, Jesús Alfredo Araujo-León ${ }^{3, *}$
${ }^{1}$ Department of Metabolism and Human Nutrition Research, Universidad Marista de Mérida, Merida, Mexico
${ }^{2}$ Nutrition Division, National Institute of Nutrition “Salvador Zubirán”, Mexico City, Mexico
${ }^{3}$ Laboratory of Chromatography, Chemistry Faculty, Universidad Autónoma de Yucatán; Merida, Mexico
*Corresponding author: jesus.araujo@correo.uady.mx

Received September 15, 2019; Revised October 21, 2019; Accepted October 26, 2019

\begin{abstract}
The vegan diet excludes animal-derived product consumption and has been linked with cardiovascular benefits. The objective of the present comparative pilot study was to analyze dietary lipid intake, evaluate the conventional clinical lipid profile, extract fatty acids from triglycerides' molecules among Mexican individuals with prolonged exposure to a vegan diet ( $\geq 3$ years), and compare these outcomes with omnivore controls. A case-control and cross-sectional comparative pilot study was performed between 2016 and 2017. Vegans were paired in a 1:1 ratio with omnivores from Merida, Mexico. A 144-item Semi-Quantitative Food Frequency Questionnaire was conducted to evaluate eating patterns. Serum fatty acids were determined from total blood with a gas chromatography assay. Lower total cholesterol, stearic, arachidonic, and trans fatty acids intake ( $p<0.001,0.014,<0.001,0.009$ respectively), but higher consumption of lauric acid were observed in the vegan group $(p=0.007)$. Vegan-diet consumers also presented greater triglycerides concentrations $(p=0.049)$; however, when analyzing fatty acids, we identified lower levels of stearic, oleic, linoleic, and arachidonic acids ( $p=0.002,0.030,0.015,0.008$, respectively). Following a vegan diet for more than three years generate modifications in serum concentrations of saturated and polyunsaturated $\omega$-6 fatty acids, which could lower inflammatory markers' biosynthesis. Potential benefits regarding cardiovascular risk may be assumed in favor of vegan individuals.
\end{abstract}

Keywords: cardiovascular risk factors, feeding patterns, lipids, nutritional status, vegan diet

Cite This Article: Alan Espinosa-Marrón, Hugo Laviada-Molina, Angélica Moreno-Enríquez, Irving F. Sosa-Crespo, Fernanda Molina-Segui, María Fernanda Villaseñor-Espinosa, José Antonio Ciau-Mendoza, and Jesús Alfredo Araujo-León, "Serum Fatty Acids Chemical Characterization after Prolonged Exposure to a Vegan Diet.” Journal of Food and Nutrition Research, vol. 7, no. 10 (2019): 742-750. doi: 10.12691/jfnr-7-10-8.

\section{Introduction}

The vegan diet entirely excludes animal-derived products consumption. [1] Following this eating pattern could implicate protection against hypertension and ischemic heart disease due to favorable modulations in blood lipids. Lower concentrations of serum lipoproteins, saturated fatty acids (SFAs), polyunsaturated fatty acids (PUFAs) $\omega-3$ and $\omega-6$ (particularly arachidonic acid), yet higher monounsaturated fatty acids' (MUFAs) levels had been reported in vegans when compared with meat-eaters. [2-5]

The ideal serum relationship between PUFAs $\omega-6 / \omega-3$ remains in a 3:1 ratio. [6] An alteration in this proportion may promote inflammation. [6,7] Arachidonic acid is a precursor of potent pro-inflammatory mediators; thus, a possible reduction in serum concentrations of this $\omega-6$ fatty acid among vegans may implicate less biosynthesis of 2-series thromboxanes, 2-series prostaglandins, and 4-series leukotrienes. [6,8] Furthermore, decreased levels of SFAs could also modulate favorably low-density lipoprotein cholesterol (LDL-C) biosynthesis. [9] These possible differences in the concentrations of lipids between plant-base diet consumers and meat-eaters could implicate diverse risk factors for cardiovascular diseases, and health advantages associated with serum lipids may be assumed in favor of vegans. [3,4,5,10]

However, the Mexican diet typically differs from other regions of the world; consequently, even when a vegan diet is followed, different physiological results may be expected due to local and cultural adaptations. [11] Plant-based diets are not entirely described among the Mexican population and it is pertinent to evaluate health's effects in this unexplored community. Inexistent information regarding serum fatty acids in vegans from Mexico addresses importance to analyze the behavior of serum lipids in this community. 


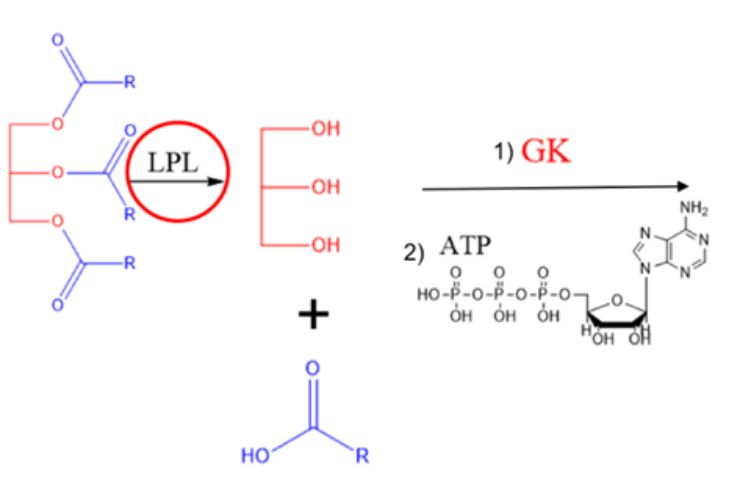

LPL: Lipoprotein lipase GK: Glicerol kinase GPO: glycerol phosphate oxidase POD: Peroxidase

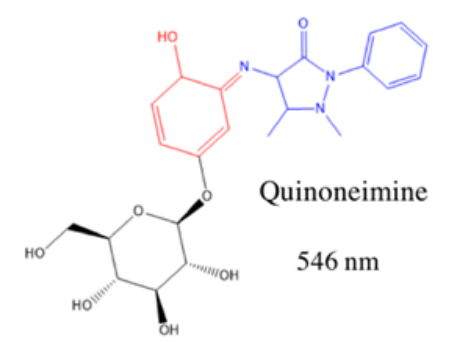

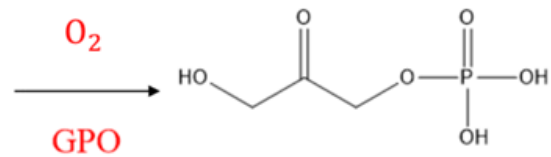

Dihydroxyacetone phosphate<smiles>CCC(C)COC(C)(C)C</smiles>

满 Glycerol 3-phosphate $\mathrm{H}_{2} \mathrm{~N}$

1)

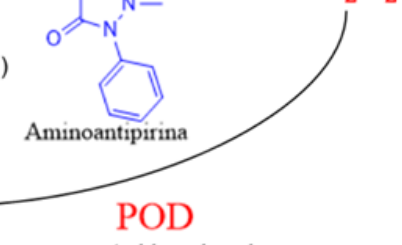

2) 4-chlorophenol

3)

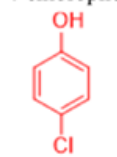

Figure 1. Triglycerides determination by spectrophotometric techniques

It should be noticed, however, that clinical techniques to determine serum triglycerides typically uses a colorimetric method by spectrophotometric technique; this methodology uses a lipase enzyme to cut the triglycerides after separating the glycerol and three mols of fatty acids, and only uses the glycerol to quantify triglycerides molecules as described in Figure 1. Nonetheless, it is relevant to classify the fatty acids that are integrated into triglycerides' structure by spectrophotometric approaches, to generate more information regarding blood lipids. Therefore, we aimed to evaluate not only the conventional clinical lipid profile, but extract fatty acids from triglycerides' molecules among Mexican individuals with prolonged exposure to a vegan diet ( $\geq 3$ years). We also compared the concentrations of fatty acids (from C14:0 [lauric acid] to C20:4 [arachidonic acid]) between plant-based diet consumers vs. omnivore controls and described them as biomarkers of cardiovascular health. We hypothesized that the local vegan diet derives into variations in serum lipoproteins and fatty acids' concentrations when compared with non-vegans, which supposes improvements in cardiovascular biomarkers.

\section{Materials and Methods}

\subsection{Study Design}

A case-control cross-sectional pilot study was conducted in south-eastern Mexican adults (Merida, Mexico) in 2017, following pre-established criteria for an adequate pilot study design and similar previously published studies. $[12,13]$ The present work adhered to the Strengthening the Reporting of Observational Studies in Epidemiology statement for case-control studies and received approval by the Ethics Committee from Universidad Marista de Merida number CE_UMM002A_2017, following the regulations regarding research protocols by Secretary of Health of Mexico (NOM-012-SSA3-2012) and Declaration of Helsinki.

\subsection{Participants}

Sixty-eight subjects from a local vegan-vegetarian group were originally identified through printed and electronic advertisement, twenty-five of them met the inclusion criteria, and twelve individuals provided written informed consent to participate in the study. Inclusion criteria contemplated female and male healthy individuals with a vegan diet for an uninterrupted period of $\geq 3$ years. They were randomly paired with controls in a 1:1 ratio. Each control was assigned arbitrarily from a triple-shortlist of candidates with an omnivore diet, considering equal gender and socioeconomic status, similar age ( \pm 5 years), and body mass index $\left( \pm 1 \mathrm{~kg} / \mathrm{m}^{2}\right)$. Controls also signed informed consent. Exclusion criteria contemplated high-performance athletes (we also verified that physical activity did not reach intensities prescribed for therapeutic purposes), those with a previous diagnosis of chronic disease, chronic alcoholism (Alcohol Use Disorder Identification Test score $>8$ ), pregnant and lactating women. Users of medications (particularly absorption-inhibition or modifiers of lipid metabolism drugs) or variations in sleep patterns that could influence variables of interest were excluded. Volunteers who followed a partial vegetarian diet were not considered either. Twenty-four participants (vegans and omnivores combined) were included in the study.

\subsection{Dietary Analysis}

A previously validated 144-item Semi-Quantitative Food Frequency Questionnaire [14] was conducted to assess monthly eating patterns in both dietary groups and to ensure full exclusion, consciously and unconsciously, of animal-derived products in the vegan sample. Daily nutrients intake was calculated by blinded nutritionists through The Food Processor Software ${ }^{\circledR}$ (ESHA research) Version 10.15.41. Healthcare professional aid regarding diet planning or supplementation habits and other health-related behaviors were also assessed in both groups. 


\subsection{Anthropometric Assessment}

For paring performance, body mass index (BMI) was determined. A previously calibrated Tanita BC-418 Segmental Body Composition Analyzer ${ }^{\circledR}$ scale was used for weight measurement, following the protocol proposed by Khalil and colleagues. [15] For height evaluation a SECA stadiometer (model 700) was used following the methods established by the International Society for the Advancement of Kinanthropometry. [16]

\subsection{Serum Lipid Assessment}

\subsubsection{Lipoproteins and Triglycerides}

Five milliliters were collected from total blood in SST tubes (without anticoagulant) under eight-hours fasting conditions. Total cholesterol (CT), low-density lipoprotein cholesterol (LDL-C), high-density lipoprotein cholesterol (HDL-C) and triglycerides (TG) were immediately determined by blinded-professionals through analytic chemistry. Cutoff points used were those stipulated by the Adult Treatment Panel III. [17] For serum TC measurement, a Trinder enzymatic method was used, which evaluates the activity of Cholesterol-Esterase-Oxidase-Peroxidase enzyme, using enzymatic endpoint method with a Randox ${ }^{\circledR}$ cholesterol reagent (CHOL) (cat ch201). The analysis was performed spectrophotometrically at 546 nanometers (nm) with a Vital Scientific analyzer (Selectra XL). HDL-C was determined by a direct method of immunoinhibition, using a Vital Scientific Selectra XL equipment, calibrated at two points with a $578 \mathrm{~nm}$ filter and Randox ${ }^{\circledR}$ reagents. For TG quantification, an enzymatic hydrolysis lipase-reaction was carried out. Total activity was determined in a Vital Scientific Selectra XL spectrophotometer, calibrated to the final point with a $546 \mathrm{~nm}$ filter and $\operatorname{Randox}^{\circledR}$ reagents. Friedewald formula was used to estimate LDL-C concentrations (in milligrams/deciliter), as presented in equation (1). [18]

$$
\text { LDL }- \text { C }=[(\text { TC })-(\text { HDL }- \text { C })]-\text { TG / } 5
$$

\subsubsection{Fatty Acids Assay}

Fatty acids' extraction was performed from 15 milliliters (mL) conical tubes, in which $1 \mathrm{~mL}$ of total blood was added with $1 \mathrm{~mL}$ of saturated sodium chloride solution, then sonicated in an ultrasound bath (Brason 1800, USA) for $2 \mathrm{~min}$ at $40 \mathrm{kHz}$. Following, a 300 microliters $(\mu \mathrm{L})$ of high-performance liquid chromatography (HPLC) grade methanol solution (Fermont, Mexico) was added and centrifuged at $4,500 \mathrm{rpm}$ at $4^{\circ} \mathrm{C}$. For lipid phase extraction, a liquid-liquid microextraction was carried out, adding $2 \mathrm{~mL}$ solution of methyl tert-butyl ether: n-hexane (MTBE: Hx, 1:1, v/v). The organic phase was concentrated to dryness with a rotary evaporator. Derivatization of lipid phase was carried out by adding $5 \mathrm{~mL}$ of trifluoride boron (14\% in methanol, Sigma Aldrich, USA) and refluxing for $15 \mathrm{~min}$ at $220^{\circ} \mathrm{C}$. The solution was transferred to a $25 \mathrm{~mL}$ conical tube, $10 \mathrm{~mL}$ of HPLC grade water was added (J.T. Baker, USA) and stirred. Finally, $1 \mathrm{~mL}$ of MTBE: HX solution $(1: 1, \mathrm{v} / \mathrm{v})$ was added and centrifuged at 4,500 revolutions per minute for $10 \mathrm{~min}$ at $20^{\circ} \mathrm{C}$. The quantitative fatty acid assay was performed in a $6890 \mathrm{~N}$ gas chromatograph (Agilent, San Jose, CA, USA) coupled with an MS 5973 Network using a DB-5MS fused silica capillary column $(50 \mathrm{~m} \times 0.25 \mathrm{~mm} \times 0.25 \mathrm{~mm}, \mathrm{~J} \& \mathrm{~W}$ GC Column; Agilent Technologies, USA). A $1 \mu \mathrm{L}$ solution of MTBE: HX with fatty acids was injected into pulsed splitless injection (70 psi for 2 min to vent) to elevate the sensitivity of the equipment. Helium (99.999\%) was used as carrier gas at $230 \mathrm{KPa}$. The Gas chromatography-mass spectrometer (CG-MS) oven temperature was programmed initially at $60^{\circ} \mathrm{C}$ for $5 \mathrm{~min}$ and increased to $315^{\circ} \mathrm{C}$ at $5^{\circ} \mathrm{C}$ min-1. Fatty acids' identification was performed with NIST Research Data Base.

Results were obtained as chromatogram of 37 fatty acid methyl ester (FAME) according to the gas chromatography column the main separate it is based on boiling point, for that, we observed between 10 to $35 \mathrm{~min}$ the saturated FAME, and from 40 to $60 \mathrm{~min}$ the C18 to C24 FAMEs.

\subsubsection{Gas Chromatography-Mass Spectrometer (GC-MS) Calibration and Validation Parameter}

A certified reference material (TraceCERT, Supelco USA) of 37 FAME (C6 to C24, saturated, monounsaturated, and polyunsaturated) was used to calibrate de Gas chromatography coupled to mass spectrometry, in range concentration of $0.5 \mathrm{mg} / \mathrm{L}$ to $50 \mathrm{mg} / \mathrm{L}$. In that same range of calibration, we used with a deuterated standard reference material of Myristid-d27 acid, Palmiditic-d31 acid, and Stearic-d35 acid all from Sigma-Adirch (USA). The deuterated FAMEs were used as an internal standard to fortified the samples. The validation parameters evaluate according to de International Council for Harmonization-Quality (ICH Q2A) for linearity, limit of detection (LOD), precision, and accuracy.

\subsection{Statistical Analysis}

A Shapiro-Wilk normality test was performed. Variables with a normal and non-normal distribution were compared between paired dietary groups through a two-tailed Student's $t$-test and a Wilcoxon signed-rank test, respectively. The association between dietary intake and serum concentrations of arachidonic acid was assessed with a Pearson's correlation coefficient. According to distribution, data is presented in means \pm SD and medians (25th and 75th percentiles). A $p \leq 0.05$ value was considered as significant. Statgraphics Centurion ${ }^{\circledR}$ software version XVII was used.

\section{Results}

\subsection{General Characteristics of Subjects}

A case-control and cross-sectional comparative pilot study was performed in twelve Mexican vegans who were randomly paired with omnivores controls in a 1:1 ratio. Subjects conditions did not differ significantly among groups $(\mathrm{BMI}=63.2 \pm 14.0$ vs. $58.2 \pm 10.6, p=0.445$; age $=29 \pm 9$ vs. $29 \pm 10, p=0.777$; vegans and omnivores, respectively), nor socioeconomic or gender. All individuals completed the study (six males and 
eighteen females). None of them were excluded based on missing data.

\subsection{Nutritional Data}

Dietary analysis is presented in Table 1. Plant-based diet group exhibited lower cholesterol, stearic, arachidonic, and total trans fatty acids intake, due to animal-derived products dietary exclusion. Contrarily, lauric SFA consumption was greater among vegans, associated with regular dietary inclusion of coconut oil (6.9 \pm 6.0 grams). It should be noticed, however, that vegan-diet consumers only showed daily consumption of soy oil (3.0 \pm 5.5 grams), olive oil (9.3 \pm 8.9 grams), almonds or nuts (18.5 \pm 10.4 grams), and avocado (29.3 \pm 13.5 grams) as unique vegetable oils' sources. Regular consumption of peanuts, cashews, pistachios, walnuts, and hazelnuts was not identified.

\subsection{Serum Lipoproteins Assay}

Conventional lipid profile analysis (Table 2) revealed greater triglycerides' concentration among vegans, and hypertriglyceridemia ( $\geq 150 \mathrm{mg} / \mathrm{dL}, 1.69 \mathrm{mmol} / \mathrm{L})$ was prevalent in three of them. This finding is related to excessive grain-based products and carbohydrates intake observed in vegans $(250.8 \pm 44.2$ vs. $208.9 \pm 55.4$ grams) as a replacement for animal-derived product consumption. No controls with hypertriglyceridemia were identified.

\subsection{Fatty Acids chemical characterization}

Fatty acids from TG molecules were extracted and quantified. Results are presented in Table 3. In agreement with dietary consumption, a significantly lower concentration of stearic acid was observed among vegans. Regarding PUFAs, a reduced concentration of oleic, linoleic, and arachidonic was also found in vegan-diet consumers. A positive association between arachidonic acid plasmatic concentrations and dietary intake was found ( $r=0.477, p=0.018)$. Furthermore, the concentrations of elaidic acid (the trans isomer of oleic acid) was decreased among vegans, this finding corresponds with the dietary intake of total trans fatty acids observed in plant-based diet consumers.

Table 1. Comparison of serum lipoproteins and triglycerides among subjects following a vegan diet with omnivores controls from a Mexican population

\begin{tabular}{|c|c|c|c|}
\hline Serum lipids (mg/dL) ${ }^{a}$ & Vegans $(n=12)$ & Omnivores $(n=12)$ & $p$-value ${ }^{\mathrm{b}}$ \\
\hline TC & $154.0 \pm 36.2$ & $167.4 \pm 45.8$ & 0.273 \\
\hline HDL-C & $51.9 \pm 10.9$ & $55.9 \pm 8.6$ & 0.337 \\
\hline LDL-C & $81.4 \pm 30.6$ & $96.0 \pm 36.8$ & 0.091 \\
\hline TG & $102.6 \pm 46.4$ & $76.7 \pm 36.8$ & $0.049 *$ \\
\hline
\end{tabular}

Data presented in means \pm SD

${ }^{a}$ International System of Units conversions: $1 \mathrm{mg} / \mathrm{dL}$ of TC, HDL-C and LDL-C= $0.26 \mathrm{mmol} / \mathrm{L} ; 1 \mathrm{mg} / \mathrm{dL}$ of TG= is $0.01 \mathrm{mmol} / \mathrm{L}$.

${ }^{\mathrm{b} O b t a i n e d ~ u s i n g ~ a ~ S t u d e n t ' s ~} t$-test for paired variables, considering 95\% confidence interval.

Abbreviations: $\mathrm{TC}=$ total cholesterol; HDL-C= high density lipoprotein cholesterol; LDL-C= low density lipoprotein cholesterol; TG= triglycerides.

Table 2. Daily dietary lipid intake comparison between vegan-diet consumers and omnivore controls from a Mexican population

\begin{tabular}{|c|c|c|c|}
\hline Dietary assessment & Vegans $(n=12)$ & Omnivores $(n=12)$ & $p$-value \\
\hline Total fat (g) & $61.2 \pm 19.0$ & $52.5 \pm 15.1$ & 0.259 \\
\hline Total cholesterol (mg) & $7.1(2.9-11.6)$ & 180.9 (139.4 - 221.7) & $<0.001$ \\
\hline Total SFA (g) & $15.3 \pm 6.6$ & $13.5 \pm 4.1$ & 0.408 \\
\hline Lauric (C12:0) (g) & $3.6(0.9-6.6)$ & $0.3(0.2-0.9)$ & 0.007 \\
\hline Myristic (C14:0) (g) & $1.5 \pm 1.2$ & $1.0 \pm 0.6$ & 0.151 \\
\hline Palmitic (C16:0) (g) & $5.5 \pm 1.4$ & $6.5 \pm 1.7$ & 0.128 \\
\hline Stearic (C18:0) (g) & $1.5 \pm 0.7$ & $2.4 \pm 0.7$ & 0.014 \\
\hline Total MUFA (g) & $22.0(17.9-31.2)$ & $21.8(16.1$ - 26.9) & 0.519 \\
\hline Palmitoleic (C16:1) (g) & $0.7(0.5-1.0)$ & $0.8(0.6-1.0)$ & 0.482 \\
\hline Oleic (C18:1) (g) & $20.2(16.1-30.5)$ & $18.9(12.5$ - 22.9) & 0.233 \\
\hline Total PUFA (g) & $14.5 \pm 3.5$ & $13.0 \pm 6.9$ & 0.472 \\
\hline Total $\omega-3$ PUFA (g) & $1.9 \pm 1.0$ & $1.4 \pm 0.5$ & 0.219 \\
\hline Total $\omega-6$ PUFA (g) & $12.5 \pm 3.1$ & $10.6 \pm 5.8$ & 0.296 \\
\hline Linoleic (C18:2) (g) & $12.3 \pm 3.2$ & $9.6 \pm 5.7$ & 0.158 \\
\hline Arachidonic (C20:4) (g) & $0.07 \pm 0.05$ & $0.22 \pm 0.09$ & $<0.001$ \\
\hline Total trans fatty acids (g) & $0.1(0.01-0.2)$ & $0.3(0.2-0.4)$ & 0.009 \\
\hline
\end{tabular}

According to distribution, data is presented as means \pm standard deviations or median $\left(25^{\text {th }}\right.$ percentile $-75^{\text {th }}$ percentile).

${ }^{a}$ Obtained using a two- tailed Student's $t$-test for paired variables or a Wilcoxon signed-rank test, considering 95\% confidence interval.

Abbreviations: SFA = saturated fatty acids; MUFA = monounsaturated fatty acids; PUFA = polyunsaturated fatty acids. 
Table 3. Fatty acids comparison between subjects with a vegan diet vs. omnivore controls from a Mexican population

\begin{tabular}{|c|c|c|c|c|c|}
\hline \multirow[t]{2}{*}{ Fatty acids } & \multicolumn{2}{|c|}{ Vegans $(n=12)$} & \multicolumn{2}{|c|}{ Omnivores $(n=12)$} & \multirow[t]{2}{*}{$p$-value ${ }^{a}$} \\
\hline & $(\mathrm{mg} / \mathrm{dL})^{\mathrm{b}}$ & $\%$ & $(\mathrm{mg} / \mathrm{dL})^{\mathrm{b}}$ & $\%$ & \\
\hline \multicolumn{6}{|l|}{ SFA } \\
\hline Lauric (C12:0) & $4.0 \pm 1.1$ & 2.2 & $3.6 \pm 0.6$ & 1.4 & 0.269 \\
\hline Myristic (C14:0) & $5.1 \pm 1.9$ & 3.2 & $5.1 \pm 1.3$ & 2.1 & 0.987 \\
\hline Palmitic (C16:0) & $60.7 \pm 57.9$ & 33.5 & $107.6 \pm 58.3$ & 34.3 & 0.061 \\
\hline Stearic (C18:0) & $13.0 \pm 8.8$ & 9.3 & $33.6 \pm 16.4$ & 12.3 & 0.002 \\
\hline \multicolumn{6}{|l|}{ MUFAS } \\
\hline Palmitoleic (C16:1) & $5.7 \pm 3.1$ & 3.9 & $5.4 \pm 1.6$ & 2.0 & 0.757 \\
\hline Oleic (C18:1) & $27.9 \pm 15.7$ & 20.0 & $55.5 \pm 28.1$ & 17.7 & 0.030 \\
\hline \multicolumn{6}{|l|}{ PUFAS $(\omega-6)$} \\
\hline Linoleic (C18:2) & $32.0 \pm 18.2$ & 22.1 & $76.1 \pm 39.9$ & 24.2 & 0.015 \\
\hline Arachidonic (C20:4) & $5.5 \pm 2.6$ & 3.0 & $11.7 \pm 4.9$ & 3.7 & 0.008 \\
\hline \multicolumn{6}{|l|}{ Unsaturated Trans } \\
\hline Elaidic & $4.8 \pm 2.5$ & 2.7 & $6.9 \pm 2.9$ & 2.2 & 0.030 \\
\hline Summary & $181.3 \pm 20.6$ & & $314.0 \pm 37.5$ & & 0.034 \\
\hline
\end{tabular}

* The equipment's limit of detection only allowed the observation of fatty acids from C14:0 to C20:4.

Data presented in means \pm SD.

a'Obtained using a Student's $t$-test for paired variables, considering 95\% confidence interval.

${ }^{\mathrm{b}}$ International System of Units conversions: $1 \mathrm{mg} / \mathrm{dL}$ of free fatty acids $=0.04 \mathrm{mmol} / \mathrm{L}$.

Abbreviations: SFA = saturated fatty acids; MUFA = monounsaturated fatty acids; PUFA = polyunsaturated fatty acids

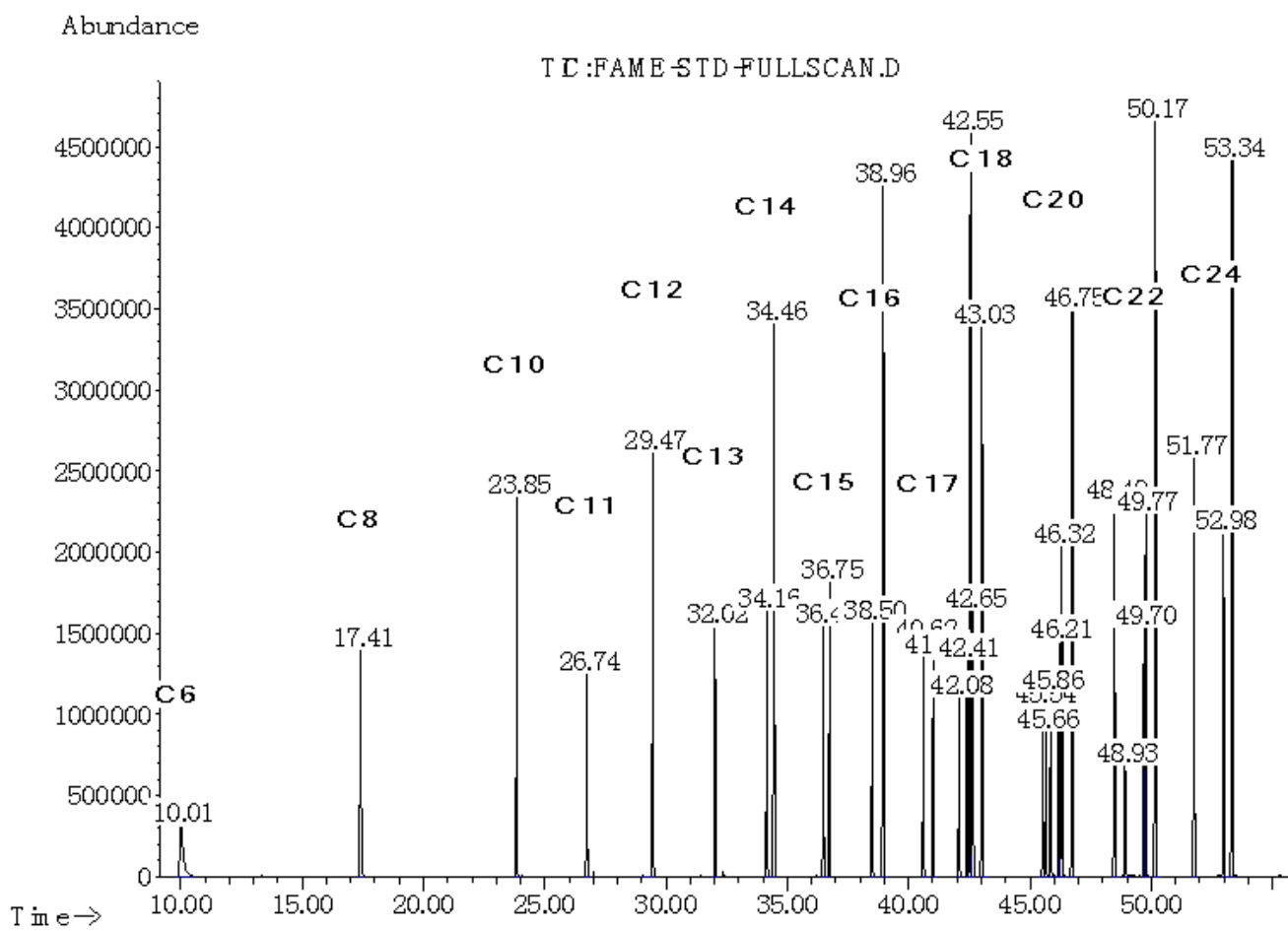

Figure 2. Chromatogram by CG-MS at $50 \mathrm{mg} / \mathrm{L}$ of 37 FAMEs

Figure 2 presents the chromatogram of 37 FAMEs, in which a good resolution between each signal and high selectivity are observed, including relevant fatty acids such as $\alpha$-linoleic acid, eicosapentaenoic acid, and docosahexaenoic acid, among other $\omega-6$ fatty acids. Our methodology and results met with the parameters established with the ICH Q2A as follows: linearity range between $0.5 \mathrm{mg} / \mathrm{L}$ to $50 \mathrm{mg} / \mathrm{L}$, all signals showed a proportional increase to the concentration with a correlation coefficient more than 0.99 , with a precision less than $1.5 \%$ and recovery percent more than $93.5 \%$ on fortified blood samples with FAMEs and deuterated FAMEs.

\section{Discussion}

A comparative analysis was conducted between individuals who follow a vegan and omnivore diet to evaluate possible variations in serum lipoproteins, TG, and fatty acids, as potential indicators of cardiovascular 
risk. Despite a lower cholesterol intake among the plant-based diet sample, no difference between groups was observed in TC serum concentrations. Even without statistical significance, two non-vegan individuals presented TC values $\geq 240 \mathrm{mg} / \mathrm{dL}(\geq 6.24 \mathrm{mmol} / \mathrm{L})$, which represent a higher cardiovascular risk as stipulated by the Adult Treatment Panel III. [17] No hypercholesterolemia was reported among vegan-diet consumers. In this sense, Wang and colleagues [19] carried out a systematic review and meta-analysis which concluded that vegetarian diets effectively reduce TC and LDL-C concentrations, being a useful non-pharmaceutical intervention to control hypercholesterolemia. Although vegan diets have been associated with blood lipids concentration's reduction when compared with meat-eaters, [19] there is discrepant evidence founding that this eating pattern does not always generate favorable changes in serum lipids. [20] In this sense, our statistical analysis indicated that a vegan diet did not significantly modify serum lipoproteins when compared with controls. It should be notice, that blood lipids can also be influenced by confounding factors different than diet- that generally distinguish vegans' lifestyle: regular practice of physical activity, management of stress through meditation techniques, support groups that could improve the attachment to a healthier lifestyle, and a reduction of alcohol and tobacco consumption are some examples. [21,22,23] However, these beneficial lifestyle factors are not necessarily widespread, since we did not identify those health-related behaviors among the vegan sample. Variations in vegans' food selection in different populations could also lead to diverse physiological results, [11] due to the different availability of food within regions, as well as cultural adaptations of the vegan diet.

We identified an imbalanced distribution of macronutrients among the plant-based diet consumers, associated with larger grain-based product consumption as a substitution for meat, which implies greater carbohydrates daily intake with potential metabolic adverse effects, such as hypertriglyceridemia. In agreement with our results, other studies have reported that vegetarians have higher TG concentrations, as well as lower levels of HDL-C, increased systolic pressure and higher prevalence of antihypertensive drug consumption compared to an omnivore population. $[11,24]$ The authors also attributed these results to changes in dietary macronutrient distribution, which supposes an excessive intake of carbohydrates as a substitution method for animal-derived products. [11] A direct relationship has been reported between environmental and sociocultural factors around the Mexican diet with dyslipidemia (high TG and low HDL- C). [25] These factors may also be affecting local vegan-diet consumers. Nevertheless, our statistical result for TG may be interpreted with caution.

Contrary to conventional plant-based dietary recommendations (frequent and vast intake of vegetable oils present in flaxseed, canola oil, walnuts, chia [Salvia hispanica] or hemp seeds, and key supplements), $[26,27,28]$ our findings only showed a feeble intake of nuts, olive oil, and avocado as sources of vegetable oils in the vegan group. No $\omega-3 / \omega-6$ PUFA supplement consumers were identified, and professional nutritional guidance was reported by only two vegan participants. In countries consuming different types and proportions of vegetable oils, vegans may likely show different serum fatty acids concentrations. [29] Consequently, sociocultural factors and food availability around the Mexican vegan diet could limit the benefits reported in other latitudes regarding serum lipids.

The main outcome of our study is that the prolonged exposure to the local plant-based diet generates modifications in the triglycerides' fatty acid profile, which imply a reduction in absolute fatty acid concentrations, with an emphasis in stearic, oleic, linoleic, arachidonic, and elaidic acids when compared to omnivore controls ( $p=0.002,0.030,0.015,0.008,0.030$, respectively). These findings coincide partially with what has been previously reported. A substantial reduction in absolute serum fatty acids levels -especially in stearic, oleic, linoleic, arachidonic, palmitic, and palmitoleic- have been shown among vegetarians when compared with omnivores. [30]

Literature reports that vegans have lower plasma concentrations of SFAs, which could represent a protective factor against cardiovascular events. [6] These findings correspond with our results, where a lower intake and serum concentration of stearic acid were found. SFAs are linked to LDL-C biosynthesis and inflammatory processes which contribute to the development of cardiometabolic. [9] However, a recent systematic review and meta-analysis performed by Chowdhury and colleagues [31] has questioned the recommendations regarding the reduction of SFAs consumption to decrease cardiovascular risk. This is a topic of current scientific debate.

Some evidence report that vegans have higher serum MUFAs' levels than non-vegan individuals. [5] Contrarily, we identified less oleic acid concentrations in vegans, and MUFAs dietary intake did not present variations between groups. In agreement with our results, similar studies had also identified lower serum oleic acid concentrations in vegetarian-diet consumers. [30] Based on these findings, we could attribute the differences in food selection between regions of the world, since we did not identify a vast quantity of MUFAs' food sources in the vegan group, such as peanuts, cashews, almonds, pistachios, macadamia, or hazelnuts. [32] Thus, benefits regarding the cholesterollowering effect may not be expected in this vegan community. [33]

Regarding PUFAs, we found decreased arachidonic and linoleic acids' concentrations in vegans' TG molecules. Individuals following a plant-based diet typically have lower serum concentrations of arachidonic acid due to animal-derived products consumption avoidance, which vastly contain in this $\omega-6$ fatty acid. [34,35] Opposing to our findings, other authors have reported through serum determination of fatty acids by gas chromatography, an increased concentration of linoleic acid in vegans $(p<0.001)$. [29] However, arachidonic acid can be synthesized through an enzymatic process of elongation and desaturation from linoleic acid. Therefore, despite having a higher linoleic intake, vegans rely on this biochemical pathway to synthesize arachidonic acid; thus, serum concentrations of both PUFAs in this sample are expected to be lower than omnivore controls. [34,36,37].

The reduction in the concentration of $\omega-6$ fatty acids can limit the biosynthesis of 2-series prostaglandins (PGE2, PGI2, PGD2, PGF2 $\alpha$ ), 2-series thromboxanes (TXA2, TXB2), 4-series leukotrienes (LTA4, LTB4, LTC4, 
LTD4, LTE4) and other proinflammatory or vasoconstriction factors (IL-1 $\beta$, IL-6 and TNF- $\alpha$ ), which contribute to the development and progression of cardiovascular diseases. $[6,38]$ By having statistically diminished the concentration of arachidonic acid, vegan participants may have fewer inflammatory factors, which could imply a lower risk of developing cardiovascular complications.

In the context of trans-fatty acids, we identified both lower intake and decreased serum concentrations of elaidic acid in vegan participants. Sarter and colleagues [35] coincide with our results, by reporting a reduction of plasma trans-fatty acids (18:1 and 18:2 isomers) in vegan-diet consumers. The authors attributed this outcome to a lower intake of products containing partially hydrogenated oils in vegans. A systematic review and meta-analysis conducted by Huang and colleagues [39] associated large intakes of trans-fatty acids with a greatest risk for developing cardiovascular diseases, the authors found a $16 \%$ lower mortality rate from circulatory diseases in vegetarians when compared with omnivores. Thus, benefits associated with positive modulations of trans-fatty serum concentrations may be extrapolated in favor of our vegan sample.

Evidence shows some results that contrast with what was found in our study. However, eating habits in Mexico are culturally and socially inequivalent to other countries, consequently, even when a plant-based diet is followed, different physiological results may be expected due to local adaptations. Individuals who follow a vegan diet typically present a regular consumption of a variety of vegetables, fruits, grains, legumes, and nuts. [3] However, these ideal patterns were not repeated strictly in this Mexican population.

This study provides pertinent evidence to the Mexican population regarding the relationship between the vegan diet and serum fatty acids modifications. Our preliminary results coincide partially with what has been reported in limited international literature. This reinforces the hypothesis that a vegan diet may imply lower concentrations of stearic, oleic, linoleic, arachidonic, and elaidic acids. However, we recognize sample size with unequal numbers of male and female participants as a limitation. Nevertheless, it is considered appropriate that a minimum of 12 subjects per group be included for pilot studies -such as the present research- based on the feasibility and precision around the estimates to be used to design future studies. [13,40] Although a full-scan was performed and 37 FAMEs were measured, some fatty acids were not expressed in grams, because they were found in quantities below the equipment's LOD.

We used a dietary tool that specifically asses "fats and oils" in one of the nine food categories. It was validated in a Latin-American country in the Spanish language, which was easily understood by our participants and allowed us to accurately collect representative data relevant for our main outcome. Furthermore, our dietary findings and novel technique for the determination of fatty acids obtained directly from TG molecules in this unexplored population are relevant to international literature and valuable for clinical practitioners, which promotes further larger research regarding this matter. Future investigations may also consider a prospective randomized trial design, to diminish the expected bias of the transversal and observational nature of our study. The cardiovascular biomarkers modifications observed after the prolonged exposure to a plant-based diet befell in normal-weight healthy young adults; nonetheless, the inclusion of participants outside normal parameters and the usage of a technique that allows a full spectrum fatty acid quantification may also be taken into consideration for future projects. The preliminary results of the present study enable that task, by presenting innovative findings that can be translated into clinical practice and proposing additional research as a complementary biochemical screening in vegan individuals.

\section{Conclusions}

This study incorporates a quantitative extraction of fatty acids from TG molecules, providing a chemical characterization which contributes to precisely depict modifications of serum lipids induced by the exposure to a vegan diet.

Vegan-diet consumers presented lower stearic and arachidonic serum concentrations, which could favorably modulate the biosynthesis of LDL-C, prostaglandins, thromboxanes, and leukotrienes with proinflammatory effects related to cardiovascular diseases. Therefore, potential benefits regarding cardiovascular risk could be in favor of those Mexican individuals who follow a vegan diet. Nonetheless, we observed that the local vegan diet may differ from other regions due to cultural adaptations. Consequently, reduced concentrations of linoleic and oleic acid were found. Hypertriglyceridemia produced by an excessive carbohydrates consumption may decrease the expected benefits in cardiovascular biomarkers.

The analysis by gas chromatography coupled with mass spectrometer generated precise information regarding serum lipids and can be applied to characterize the FAME in blood samples with excellent and safe results that can be employed in future research.

\section{Acknowledgments}

This work was funded by Chemistry faculty by grant "SISTPROY FQUI-2016-0005” with name "Comparación de los perfiles cromatográficos de ácidos grasos presentes en la sangre de pacientes con normopeso, sobrepeso y obesos, como herramienta en el diagnóstico clínico de la hipertrigliceridemia”. We thank our colleagues BSc. Jenny Maldonado-Molina and BSc. Abril Paulina Arroyo-Méndez for data collection contribution, and MSc. Jaidy ChávezMedia for final advising. Finally, all authors we grateful with Universidad Marista, National Institute of Nutrition "Salvador Zubirán" and Chemistry Faculty from Universidad Autónoma de Yucatán for the facilities in the use of the departments, scientific equipment, and software for the development the investigation.

\section{Conflict of Interests}

The authors have no competing interests. 


\section{List of Abbreviations}

MTBE= methyl tert-butyl ether

FAME $=$ fatty acid methyl ester

ICH Q2A= International Council for HarmonizationQuality.

\section{References}

[1] Clarys, P., Deliens, T., Huybrechts, I., Deriemaeker, P., Vanaelst, B., De Keyzer, W., Hebbelinck, M., Mullie, P., "Comparison of nutritional quality of the vegan, vegetarian, semi-vegetarian, pesco-vegetarian and omnivorous diet", Nutrients, 6(3): 1318-1332, March 2014.

[2] Burdge, G.C., Tan, S-Y., Henry, C.J., "Long-chain n-3 PUFA in vegetarian women: a metabolic perspective”, J Nutr Sci, 6: 1-8. November 2017.

[3] Melina, V., Craig, W., Levin, S, "Position of the Academy of Nutrition and Dietetics: Vegetarian Diets”, J Acad Nutr Diet, 116(12): 1970-1980. December 2016.

[4] Dewell, A., Weidner, G., Sumner, M.D., Chi, C.S., Ornish, D., “A Very-Low-Fat Vegan Diet Increases Intake of Protective Dietary Factors and Decreases Intake of Pathogenic Dietary Factors”, $J$ Am Diet Assoc, 108(2):347-356. February 2008.

[5] Benatar, J., Stewart, R, “Cardiometabolic Risk Factors and Plasma Fatty Acids in Vegans - Results of an Observational Study”. Hear Lung Circ, 26: S344. 2017.

[6] Catalán, J.S., Agüero, S.D., García, J.T, “Los ácidos grasos dietarios y su relación con la salud”, Nutr Hosp, 32(3): 1362-1375. September 2015.

[7] Wood, K.E., Mantzioris, E., Gibson, R.A., Muhlhausler, B.S, "Incorporating macadamia oil and butter to reduce dietary omega6 polyunsaturated fatty acid intake”, Nutr Diet, 70:94-100. June 2013.

[8] Innes, J.K., Calder, P.C, "Prostaglandins, Leukotrienes and Essential Fatty Acids Omega-6 fatty acids and inflammation”, Elsevier, Plefa (Prostaglandins, Leukot Essent Fat Acids) 7: 1-8. May 2018.

[9] Bier, D.M, "Saturated Fats and Cardiovascular Disease: Interpretations Not as Simple as They Once Were”, Crit Rev Food Sci Nutr, 56(12): 1943-1946. September 2016.

[10] Harris, W.S, "Achieving optimal n-3 fatty acid status: the vegetarian's challenge . . . or not”, Am J Clin Nutr, 100(1): 449-452. June 2014.

[11] Sun, C., Hsu, H., Chen, S, "Vegetarian diet is associated with higher SBP and fasting serum triacylglycerol than omnivorous diet in Taiwanese type 2 diabetic", Diabetes Res Clin Pract, 120: S141. October 2016.

[12] Foster, R.L, "What a pilot study is and what it is not", $J$ Spec Pediatr Nurs, 18(1): 1-2. January 2013.

[13] Rogerson, D., Maçãs, D., Milner, M., Liu, Y., Klonizakis, M., "Contrasting effects of short-term mediterranean and vegan diets on microvascular function and cholesterol in younger adults: A comparative pilot study”, Nutrients, 10(12). December 2018.

[14] Monsalve Álvarez, J.M., González Zapata, L.I, “Diseño de un cuestionario de frecuencia para evaluar ingesta alimentaria en la Universidad de Antioquia, Colombia”, Nutr Hosp, 26(6): 1333-1344. November 2011.

[15] Khalil, S.F., Mohktar, M.S., Ibrahim, F, “The theory and fundamentals of bioimpedance analysis in clinical status monitoring and diagnosis of diseases", Sensors (Switzerland) 14(6): 10895-10928. June 2014.

[16] Stewart, A.D., Marfell-Jones, M., Olds, T., International Standards for Anthropometric Assessment, International Society for the Advancement of Kinanthropometry, Wellington, 2001.

[17] National Institute of Health, "NCEP Cholesterol Guidelines. [NCEP]” Natl Cholest Educ Progr ATP III, 329: 925-929. May 2001.

[18] Martin, S.S., Blaha, M.J., Elshazly, M.B., Toth, P.P., Kwiterovich, P.O., Blumenthal, R.S., Jones, S.R., "Comparison of a novel method vs the Friedewald equation for estimating low-density lipoprotein cholesterol levels from the standard lipid profile”, JAMA - J Am Med Assoc, 310: 2061-2068. November 2013.

[19] Wang, F., Zheng, J., Yang, B., Jiang, J., Fu, Y., Li, D., "Effects of vegetarian diets on blood lipids: A systematic review and meta-analysis of randomized controlled trials”, J Am Heart Assoc, 4(10): e002408. October 2015.

[20] Sanasam, M., Shashikiran H.C., Prashanth, S., Ganesh P., Chandrakanth, K.K., "Effect of a plant versus animal based diet on lipid profile of yoga practicing medical students: a pilot study.” Int J Res Med Sci, 6:1207. November 2018.

[21] Shatwan, I.M., Winther, K.H., Ellahi, B., Elwood, P., Ben-Shlomo, Y., Givens, I., Rayman, M.P., Lovegrove, J.A., Vimaleswaran, K.S., "Association of apolipoprotein e gene polymorphisms with blood lipids and their interaction with dietary factors”, Lipids Health Dis, 17: 1-14. April 2018.

[22] Latha Marupudi, M., Rao Gondi, S., Sesha Sai Krishna Manne V, Yalavarti S, "Confounding Effects of Age, Diet and Physical Activity on Blood Pressure”, J Evol Med Dent Sci, 5(34): 1888-1891. April 2017.

[23] Medina, C., Janssen, I., Barquera, S., Bautista-Arredondo, S., González, M.E., González, C, "Occupational and leisure time physical inactivity and the risk of type II diabetes and hypertension among Mexican adults: A prospective cohort study”, Sci Rep, 8(1): 4-10. March 2018.

[24] Kim, M.K., Cho, S.W., Park, Y.K. "Long-term vegetarians have low oxidative stress, body fat, and cholesterol levels”, Nutr Res Pract, 6(2): 155-161. April 2012.

[25] Méndez-Hernández, P., Dosamantes-Carrasco, L.D., Siani, C., Pierlot, R., Martínez-Gómez, M., Rivera-Paredez, B., CervantesPopoca, L., Rojas-Lima, E., Salazar-Martínez, E., Flores, Y.N., Salmerón, J., "Mealtime habits and risk of developing the metabolic syndrome or insulin resistance among Mexican adults", Br J Nutr, 116: 1824-1833. November 2016.

[26] Kim, S., Fenech, M.F., Kim, P.J, "Nutritionally recommended food for semi- to strict vegetarian diets based on large-scale nutrient composition data”, Sci Rep, 8: 1-11. March 2018.

[27] Saini, R.K., Keum, Y.S, “Omega-3 and omega-6 polyunsaturated fatty acids: Dietary sources, metabolism, and significance -A review”, Life Sci 15(203): 255-267. June 2018.

[28] McGirr, C., McEvoy, C.T., Woodside, J. V, Vegetarian and Vegan Diets: Weighing the Claims. In: Nutrition Guide for Physicians and Related Healthcare Professionals, Springer International Publishing, Cham, 2017, 203-212.

[29] Elorinne, A.L., Alfthan, G., Erlund, I., Kivimäki, H., Paju, A., Salminen, I., Turpeinen, U., Voutilainen, S., Laakso, J., "Food and nutrient intake and nutritional status of Finnish vegans and nonvegetarians”, PLoS One, 11(2): 1-14. February 2016.

[30] Kirkeby, K., Bjerkedal, I, "The fatty acid composition in serum of norwegian vegetarians”, Acta Med Scand, 183(1-2): 143-148. January 1968.

[31] Chowdhury, R., Warnakula, S., Kunutsor, S., Crowe, F., Ward, H.A., Johnson, L., Franco, O.H., Butterworth, A.S., Forouhi, N.G., Thompson, S.G., Khaw, K.T., Mozaffarian, D., Danesh, J., Di Angelantonio, E., "Association of dietary, circulating, and supplement fatty acids with coronary risk: A systematic review and meta-analysis”, Ann. Intern. Med, 160(6): 398-406. March 2014.

[32] Schwingshackl, L., Hoffmann, G., "Monounsaturated fatty acids, olive oil and health status: a systematic review and meta-analysis of cohort studies”, Lipids Health Dis, 13: 154. October 2014.

[33] Calder, P.C, "Functional Roles of Fatty Acids and Their Effects on Human Health”, J Parenter Enter Nutr, 39 (1 Suppl): 18S-32S. July 2015.

[34] Saunders, A. V., Davis, B.C., Garg, M.L, “Omega-3 polyunsaturated fatty acids and vegetarian diets”, Med J Aust, 199(S4): S22-6. August 2013.

[35] Sarter, B., Kelsey, K.S., Schwartz, T.A., Harris, W.S, "Blood docosahexaenoic acid and eicosapentaenoic acid in vegans: Associations with age and gender and effects of an algal-derived omega-3 fatty acid supplement”, Clin Nutr, 34(2): 212-218. March 2015.

[36] Kothapalli, K.S.D., Ye, K., Gadgil, M.S., Carlson, S.E., O’Brien, K.O., Zhang, J.Y., Park, H.G., Ojukwu K., Zou, J., Hyon, S.S., Joshi, K.S., Gu, Z., Keinan, A., Brenna, J.T., "Positive Selection on a Regulatory Insertion-Deletion Polymorphism in FADS2 Influences Apparent Endogenous Synthesis of Arachidonic Acid”, Mol Biol Evol, 33(7): 1726-1739. March 2016. 
[37] Greupner, T., Kutzner, L., Nolte, F. Strangmann, A., Kohrs, H., Hahn, A., Schebb, N.H., Schuchardt, J.P., "Effects of a 12-week high- $\alpha$-linolenic acid intervention on EPA and DHA concentrations in red blood cells and plasma oxylipin pattern in subjects with a low EPA and DHA status", Food Funct, 9(3): 1587-1600. March 2018.

[38] Patterson, E., Wall, R., Fitzgerald, G.F., Ross, R.P., Stanton, C., "Health Implications of High Dietary Omega-6 Polyunsaturated Fatty Acids”, J Nutr Metab, 2012: 1-16. April 2012.
[39] Huang, T., Yang, B., Zheng, J., Li, G., Wahlqvist, M.L., Li, D., "Cardiovascular Disease Mortality and Cancer Incidence in Vegetarians: A Meta-Analysis and Systematic Review" Ann Nutr Metab, 60(4): 233-240. June 2012.

[40] Julious, S.A., "Sample size of 12 per group rule of thumb for a pilot study”, Pharm Stat, 4: 287-291. November 2005. 